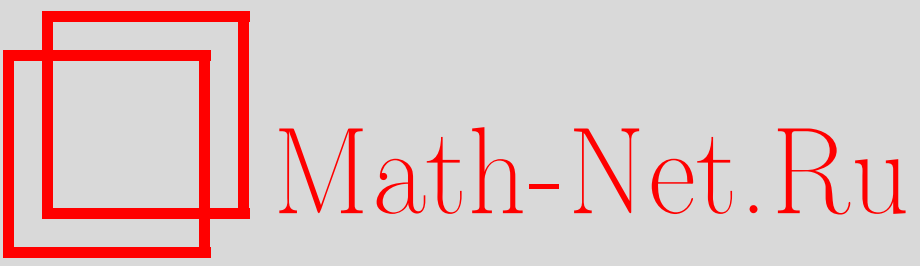

В. П. Радченко, А. А. Усов, Модификация сеточных методов решения линейных дифференциальных уравнений с переменными коэффициентами на основе тейлоровских разложений, Вестн. Сам. гос. техн. ун-та. Сер. Физ.-мат. науки, 2008, выпуск 2(), 60-65

DOI: https://doi.org/10.14498/vsgtu646

Использование Общероссийского математического портала Math-Net.Ru подразумевает, что вы прочитали и согласны с пользовательским соглашением http: //www . mathnet.ru/rus/agreement

Параметры загрузки:

IP : 54.196 .121 .252

26 апреля 2023 г., 17:58:12 
УДК 517.927:519.624

\section{МОДИФИКАЦИЯ СЕТОЧНЫХ МЕТОДОВ РЕШЕНИЯ ЛИНЕЙНЫХ ДИФФЕРЕНЦИАЛЬНЫХ УРАВНЕНИЙ С ПЕРЕМЕННЫМИ КОЭФФИЦИЕНТАМИ НА ОСНОВЕ ТЕЙЛОРОВСКИХ РАЗЛОЖЕНИЙ}

\section{В. П. Радченко, А. А. Усов}

Самарский государственный технический университет, 443100, Самара, ул. Молодогвардейская, 244.

E-mail: radch@samgtu.ru

Предложен модифицированный сеточный метод решения краевых задач для линейных дифберенииальных уравнений на основе тейлоровских разложений. Показано, что точность предложенного метода на несколько порядков выше классического метода сеток. Приведены резулътаты численных экспериментов.

Ключевые слова: обыкновенные дифференциалъные уравнения, сеточный метод, тейлоровские разложения, точность, погрешность.

Введение. Несмотря на большие успехи в теории дискретных численных методов решения обыкновенных дифференциальных уравнений (ДУ), и в настоящее время проявляется неослабевающий интерес к разработке новых и модификации существующих численных методов решения дифференциальных уравнений с точки зрения повышения точности результатов при минимизации количества вычислительных процедур. Как известно, классические методы основаны на аппроксимации соответствующих производных через конечные разности и замене дифференциального оператора на разностный. Одним из самых простых и доступных сеточных способов построения решений ДУ является метод тейлоровских разложений. Однако он в основном применяется лишь для решения задач Коши (например, [1-5]) и хорошо зарекомендовал себя при построении высокоточных решений в небесной механике $[4,5]$.

В настоящей работе сделана попытка модификации существующих сеточных подходов к решению краевых задач ДУ на основе тейлоровских разложений.

Хорошо известно, что при численном решении краевых задач возникают дополнительные трудности по сравнению со случаем задачи Коши, поскольку здесь необходимо решать систему уравнений и исследовать математические особенности ее решения. Не касаясь всей широты и строгости задачи, рассмотрим предлагаемый метод на примере краевой задачи для обыкновенного линейного ДУ второго порядка с переменными коэффициентами.

1. Метод тейлоровских разложений для решения краевых задач. Рассмотрим краевую задачу:

$$
y^{\prime \prime}+p(x) y^{\prime}+g(x) y=f(x), \quad a \leqslant x \leqslant b,
$$

Радченко Владимир Павлович-заведующий кафедрой «Прикладная математика и информатика» Самарского государственного технического университета; д.ф.-м.н., профессор.

Усов Александр Александрович - инженер кафедры высшей математики и прикладной информатики Самарского государственного технического университета. 


$$
\alpha_{0} y(a)+\beta_{0} y^{\prime}(a)=\gamma_{0}, \quad \alpha_{1} y(b)+\beta_{1} y^{\prime}(b)=\gamma_{1},
$$

где $p, g, f$ - заданные функции требуемой гладкости; $\alpha_{i}, \beta_{i}, x_{i}$ - заданные числа, причём $\alpha_{i}^{2}+\beta_{i}^{2}>0(i=0,1)$. Согласно терминологии [6] в случае, если $\alpha_{i}=1, \beta_{i}=0$, краевое условие $(2)$ называется условием первого рода. Если $\alpha_{i}=0, \beta_{i}=1$, то имеем условие второго рода, а при $\alpha_{i} \neq 0, \beta_{i} \neq 0-$ краевое условие третьего рода. В дальнейшем, не нарушая общности задачи, в целях сокращения дальнейших выкладок будем рассматривать краевые условия первого рода:

$$
y(a)=\gamma_{0}, \quad \gamma(b)=\gamma_{1}
$$

В соответствии с сеточными методами введём дискретизацию области $a \leqslant x \leqslant b$ точками $a=x_{0}<x_{1}<x_{2}<\ldots<x_{n-1}<x_{n}=b$ с шагом дискретизации $h=\frac{b-a}{n}(n \in \mathbb{N}, n \geqslant 2)$, так что $x_{i+1}=x_{i}+h(i=0,1,2, \ldots, n-1)$. Значения любой сеточной функции $z=z(x)$ и её производных будем обозначать общепринятым образом: $z\left(x_{i}\right)=z_{i}, z^{\prime}\left(x_{i}\right)=z_{i}^{\prime}, z^{\prime \prime}\left(x_{i}\right)=z_{i}^{\prime \prime}, \ldots$

Для построения численного решения задачи $(1),(3)$ запишем сначала разложение функции $y=y(x)$ в ряд Тейлора в точке $x=x_{i}$, ограничиваясь вторым членом ряда:

$$
\begin{gathered}
y\left(x_{i}\right)+y^{\prime}\left(x_{i}\right)(-h)+\frac{y^{\prime \prime}\left(x_{i}\right) h^{2}}{2} \cong y\left(x_{i-1}\right) \\
y\left(x_{i}\right)+y^{\prime}\left(x_{i}\right) h+\frac{y^{\prime \prime}\left(x_{i}\right) h^{2}}{2} \cong y\left(x_{i+1}\right), \quad i=1,2, \ldots, n-1 .
\end{gathered}
$$

Кроме этого из уравнения (1) имеем

$$
g\left(x_{i}\right) y\left(x_{i}\right)+p\left(x_{i}\right) y^{\prime}\left(x_{i}\right)+y^{\prime \prime}\left(x_{i}\right)=f\left(x_{i}\right), \quad i=1,2, \ldots, n-1 .
$$

Принимая $y\left(x_{i}\right), y^{\prime}\left(x_{i}\right), y^{\prime \prime}\left(x_{i}\right)$ за неизвестные, систему трех уравнений (4)-(6) можно записать в матричной форме:

$$
M \cdot X=B
$$

где $X=\left(y_{i}, y_{i}^{\prime}, y_{i}^{\prime \prime}\right)^{\mathrm{T}}, B=\left(y_{i-1, y_{i+1}, f_{i}}\right)^{\mathrm{T}}, M=\left(\begin{array}{ccc}1 & -h & \frac{h^{2}}{2} \\ 1 & h & \frac{h^{2}}{2} \\ g_{i} & p_{i} & 1\end{array}\right)$ - матрица из коэффициентов при неизвестных $y_{i}, y_{i}^{\prime}, y_{i}^{\prime \prime} ; \mathrm{T}$ - операция транспонирования матриц.

Предполагается, что функции $g(x), p(x)$ такие, что в любой точке $x=x_{i}$ $(i=1, \ldots, n-1)$ выполняется $\operatorname{det} M \neq 0$. Тогда существует обратная матрица $M^{-1}$ к матрице $M$ в любой точке $x=x_{i}(i=1,2, \ldots, n-1)$. Обозначая элементы обратной матрицы через $m_{i j}^{-1}(i, j=1,2,3)$, найдем решение матричного уравнения (7):

$$
X=M^{-1} \cdot B
$$

или в координатной форме: 


$$
\begin{aligned}
& m_{11}^{-1}\left(x_{i}\right) y_{i-1}+m_{12}^{-1}\left(x_{i}\right) y_{i+1}+m_{13}^{-1}\left(x_{i}\right) f_{i}=y_{i}, \\
& m_{21}^{-1}\left(x_{i}\right) y_{i-1}+m_{22}^{-1}\left(x_{i}\right) y_{i+1}+m_{23}^{-1}\left(x_{i}\right) f_{i}=y_{i}^{\prime}, \\
& m_{31}^{-1}\left(x_{i}\right) y_{i-1}+m_{32}^{-1}\left(x_{i}\right) y_{i+1}+m_{33}^{-1}\left(x_{i}\right) f_{i}=y_{i}^{\prime \prime},
\end{aligned}
$$

$i=1,2, \ldots, n-1$, при этом в соответствии с (3) имеем: $y_{0}=\gamma, y_{n}=\gamma_{1}$.

Переписывая (9) в виде

$$
m_{11}^{-1}\left(x_{i}\right) y_{i-1}-y_{i}+m_{12}^{-1}\left(x_{i}\right) y_{i+1}=-m_{13}^{-1}\left(x_{i}\right) f_{i},
$$

$i=1,2, \ldots, n-1$, получаем систему $n-1$ уравнения относительно $n-1$ неизвестного $y_{i}(i=1,2, \ldots, n-1)$, которая может быть решена, например, методом прогонки. После определения величин $y_{i}(i=1,2, \ldots, n-1)$ из (10) и (11) чисто алгебраически можно найти величины производных $y_{i}^{\prime}, y_{i}^{\prime \prime}$ $(i=1,2, \ldots, n-1)$.

Можно показать, что в этом случае система уравнений (12) эквивалентна классическому методу сеток с аппроксимациями производных

$$
y_{i}^{\prime}=\frac{y_{i+1}-y_{i-1}}{2 h}, \quad y_{i}^{\prime \prime}=\frac{y_{i-1}-2 y_{i}+y_{i+1}}{h^{2}}
$$

и погрешностью решения $o\left(h^{2}\right)$. Очевидно, что преимущества метода Тейлора в этом случае нивелируются. Однако, увеличивая число членов разложения в формуле Тейлора, можно существенно увеличить точность метода, не меняя шага $h$ и шаблона сетки.

Рассмотрим теперь случай, когда в формуле Тейлора используется пять членов разложения. Дифференцируя два раза уравнение (1), получим

$$
\begin{gathered}
y^{\prime \prime \prime}+p(x) y^{\prime \prime}+\left(p^{\prime}(x)+g(x)\right) y^{\prime}+g^{\prime}(x) y=f^{\prime}(x), \\
y^{(4)}+p(x) y^{\prime \prime \prime}+\left(2 p^{\prime}(x)+g(x)\right) y^{\prime \prime}+\left(p^{\prime \prime}(x)+2 g^{\prime}(x)\right) y^{\prime}+g^{\prime \prime}(x) y=f^{\prime \prime}(x) .
\end{gathered}
$$

Из разложений функции в ряды Тейлора имеем:

$$
\begin{aligned}
& y_{i}-y_{i}^{\prime} h+\frac{h^{2}}{2 !} y_{i}^{\prime \prime}-\frac{h^{3}}{3 !} y_{i}^{\prime \prime \prime}+\frac{h^{4}}{4 !} y_{i}^{(4)} \cong y_{i-1}, \\
& y_{i}+y_{i}^{\prime} h+\frac{h^{2}}{2 !} y_{i}^{\prime \prime}+\frac{h^{3}}{3 !} y_{i}^{\prime \prime \prime}+\frac{h^{4}}{4 !} y_{i}^{(4)} \cong y_{i+1} .
\end{aligned}
$$

Тогда из соотношений (6), (14)-(17) получим систему уравнений (7), в которой векторы неизвестных $X$, свободных членов $B$ и матрица $M$ имеют вид:

$$
\begin{gathered}
X=\left(y_{i}, y_{i}^{\prime}, y_{i}^{\prime \prime}, y_{i}^{\prime \prime \prime}, y_{i}^{(4)}\right)^{\mathrm{T}}, \\
B=\left(y_{i-1}, y_{i+1}, f_{i}, f_{i}^{\prime}, f_{i}^{\prime \prime}\right)^{\mathrm{T}}, \\
M=\left(\begin{array}{ccccc}
1 & -h & \frac{h^{2}}{2} & -\frac{h^{3}}{6} & \frac{h^{4}}{24} \\
1 & h & \frac{h^{2}}{2} & \frac{h^{3}}{6} & \frac{h^{4}}{24} \\
g_{i} & p_{i} & 1 & 0 & 0 \\
g_{i} & p_{i}^{\prime}+g_{i} & p_{i} & 1 & 0 \\
g_{i}^{\prime \prime} & p_{i}^{\prime \prime}+2 g_{i}^{\prime} & 2 p_{i}^{\prime}+g_{i} & p_{i} & 1
\end{array}\right) .
\end{gathered}
$$


Вновь, предполагая $\operatorname{det} M \neq 0$, из (8) находим вектор-столбец неизвестных $X$ :

$$
\begin{aligned}
& m_{11}^{-1}\left(x_{i}\right) y_{i-1}+m_{12}^{-1}\left(x_{i}\right) y_{i+1}+m_{13}^{-1}\left(x_{i}\right) f_{i}+m_{14}^{-1}\left(x_{i}\right) f_{i}^{\prime}+m_{15}^{-1}\left(x_{i}\right) f_{i}^{\prime \prime}=y_{i}, \\
& m_{k 1}^{-1}\left(x_{i}\right) y_{i-1}+m_{k 2}^{-1}\left(x_{i}\right) y_{i+1}+m_{k 3}^{-1}\left(x_{i}\right) f_{i}+ \\
& \quad+m_{k 4}^{-1}\left(x_{i}\right) f_{i}^{\prime}+m_{k 5}^{-1}\left(x_{i}\right) f_{i}^{\prime \prime}=y_{i}^{(k-1)} \quad(k=2,3,4,5),
\end{aligned}
$$

где $i=1,2, \ldots, n-1 ; y_{i}^{(k-1)}$ - значения соответствующих производных.

Переписав (18) в виде

$$
\begin{aligned}
m_{11}^{-1}\left(x_{i}\right) y_{i-1}-y_{i}+m_{12}^{-1}( & \left.x_{i}\right) y_{i+1}=-\left(m_{13}^{-1}\left(x_{i}\right) f_{i}+\right. \\
& \left.+m_{14}^{-1}\left(x_{i}\right) f_{i}^{\prime}+m_{15}^{-1}\left(x_{i}\right) f_{i}^{\prime \prime}\right), \quad i=1, \ldots, n-1,
\end{aligned}
$$

вновь получим систему из $(n-1)$ уравнений относительно $(n-1)$ неизвестных $y_{i}, i=1,2, \ldots, n-1$; величины $y_{0}=\gamma_{0}$ и $y_{n}=\gamma_{1}$ известны из граничных условий (3). После решения системы уравнений (20) по формулам (19) чисто алгебраически могут быть найдены производные функции $y=y(x)$ до четвертого порядка включительно.

Аналогично, варьируя числом членов разложения в ряд Тейлора и дифференцируя нужное число раз исходное дифференциальное уравнение (1), можно получать более точные разностные схемы типа (19) и (20).

Прямая аналитическая оценка возникающей при этом погрешности решений для $y_{i}$ и её производных - самостоятельная и достаточно трудная задача, которая не является целью данной работы.

2. Численное исследование погрешности метода. Анализ возникающей погрешности предложенного метода решения в зависимости от числа членов разложения в ряде Тейлора выполним на следующем конкретном примере:

$$
\begin{gathered}
y^{\prime \prime}+\frac{2}{x} y^{\prime}+y=\frac{1}{x} \quad(1 \leqslant x \leqslant 9), \\
y(1)=85, \quad y(9)=4 .
\end{gathered}
$$

Общее решение дифференциального уравнения (21) имеет вид [7]

$$
y(x)=\frac{C_{1} \cos (x)+C_{2} \sin (x)+1}{x},
$$

а частное решение задачи (21), (22) задается функцией (23) при $C_{1}=5,222$; $C_{2}=96,472$.

Оценим теперь погрешности численных решений задачи (21), (22) по предлагаемому методу, полученных с разным числом членов в ряде Тейлора (до $h^{2}, h^{4}, h^{6}$ ) и различными значениями шага $h$, с точным аналитическим решением по норме

$$
\|\delta\|=\left(\frac{\sum_{i=1}^{n-1}\left(y_{i}^{p}-y_{i}^{a}\right)^{2}}{\sum_{i=1}^{n-1}\left(y_{i}^{a}\right)^{2}}\right)^{\frac{1}{2}},
$$


где $y_{i}^{p}$ - численное решение; $y_{i}^{a}$ - аналитическое решение, полученное по (23); $n$ - число разбиений отрезка $[1 ; 9]$.

По формуле, аналогичной (24), оценивались погрешности и для производных $y^{\prime}$ и $y^{\prime \prime}$.

В таблице приведены результаты сравнения решений для функции $y$ и её производных $y^{\prime}$ и $y^{\prime \prime}$, полученных численным методом с использованием тейлоровских разложений с числом членов $k=3$ (разложение до членов с $h^{2}$ ), $k=5$ (до $h^{4}$ ) и $k=7$ (до $h^{6}$ ) с точным решением (23) для функции $y=y(x)$ и её производных $y^{\prime}$ и $y^{\prime \prime}$ в зависимости от шага дискретизации $h$. Как уже отмечалось выше, решение при $k=3$ соответствует классическому методу сеток с аппроксимацией производных соотношениями (13), при этом шаблон трёхточечный. Как следует из приведенных данных, даже при столь грубом разбиении интервала интегрирования точность предлагаемого метода, например, для функции $y$ (при сохранении шага дискретизации $h$ ) уже при числе членов тейлоровского разложения $k=5$ увеличивается на порядок, а при $k=7$ - на три порядка по сравнению с классическим методом $(k=3)$. При этом, во-первых, не увеличивается размерность основной системы уравнений относительно значений $y_{i}$ (типа (21)), во-вторых, сохраняется трёхточечный шаблон, что позволяет решать систему уравнений типа (21) при разных $k$ одним и тем же методом. Аналогичные результаты получены и для производных $y^{\prime}$ и $y^{\prime \prime}$.

Увеличение разрешающей способности (по точности) при использовании классического подхода возможно двумя путями: увеличением порядка аппроксимации производных, что ведёт к увеличению узлов в шаблоне, или уменьшением шага дискретизации и связанного с этим увеличением размерности основной системы уравнений относительно узловых значений $y_{i}$. В частности, вычислительный эксперимент показал, что для достижения заданной точности решений, полученных методом тейлоровских разложений в случае $h=0,4$ и $k=7\left(\|\delta\|_{y}=9,97 \cdot 10^{-7},\|\delta\|_{y^{\prime}}=1,72 \cdot 10^{-5},\|\delta\|_{y^{\prime \prime}}=4,06 \times\right.$ $\times 10^{-5}$ ), классическим сеточным методом необходимо уменьшение шага в 200 раз, при этом достигнута лишь точность по функции $y=y(x)$, а погрешности по производным $\left(\|\delta\|_{y^{\prime}}=3,53 \cdot 10^{-4},\|\delta\|_{y^{\prime \prime}}=4,75 \cdot 10^{-4}\right)$ ниже соответствующих погрешностей по предлагаемому методу.

\begin{tabular}{|c|c|c|c|c|}
\hline & & й $y$ & $\begin{array}{l}\text { А }\|\delta\| \text { I } \\
\text { роизво }\end{array}$ & $\begin{array}{l}\text { me }(24) \\
y^{\prime} \text { и } y^{\prime \prime}\end{array}$ \\
\hline$k$ & $h$ & $y$ & $y^{\prime}$ & $y^{\prime \prime}$ \\
\hline & 0 , & 0.16 & 0,14 & 0,36 \\
\hline 3 & 0 , & 0,0 & 0,04 & 0,106 \\
\hline & 0,2 & 0,0 & 0,009 & 0,030 \\
\hline & $0, \varepsilon$ & $1,7 \cdot$ & $2,2 \cdot 10^{-2}$ & $1,5 \cdot 10^{-2}$ \\
\hline 5 & 0,4 & $1,5 \cdot 1$ & $4,8 \cdot 10^{-3}$ & $6.2 \cdot 10^{-3}$ \\
\hline & 0,2 & 2,06 . & $1,3 \cdot 10^{-3}$ & $2,58 \cdot 10^{-3}$ \\
\hline & 0 & 1 & $2,26 \cdot 10^{-4}$ & $3,56 \cdot 10^{-4}$ \\
\hline 7 & 0,4 & $9,97 \cdot 1$ & $1,72 \cdot 10^{-}$ & $4,06 \cdot 10^{-5}$ \\
\hline & 0,2 & $7,78 \cdot 10$ & $6,61 \cdot 10^{-7}$ & $1,81 \cdot 10^{-6}$ \\
\hline
\end{tabular}


3. Выводы. Таким образом, предложен метод решения краевых задач (на примере линейных дифференциальных уравнений второго порядка с переменными коэффициентами) на основе тейлоровских разложений. Результаты численных экспериментов показали, что точность разработанного метода на несколько порядков (в зависимости от числа членов разложения) выше, чем в классическом методе сеток, при этом шаг дискретизации, шаблон и (соответственно) размерность основной системы уравнений не изменяются.

Работа выполнена при финансовой поддержке Федералъного агентства по образованию (проект № РНП.2.1.1.1689).

\section{БИБЛИОГРАФИЧЕСКИЙ СПИСОК}

1. Самарский, А. А. Численные методы [Текст] / А. А. Самарский, А. В. Гулин.-М.: Наука, 1989. - 432 с. - ISBN 5-02-013996-3.

2. Самарский, A.А. Методы решения сеточных уравнений [Текст] / А.А. Самарский, Е. С. Николаев. - М.: Наука, 1978. - 591 с.

3. Бахвалов, Н. С. Численные методы [Текст] / Н. С. Бахвалов, Н. П. Жидков, Г. М. Кобельков. - М.: Лаборатория базовых знаний, 2001. - 632 с. - ISBN 5-93208-043-4.

4. Заусаев, А.Ф. Дискретные численные методы решения обыкновенных дифференциальных уравнений [Текст] / А.Ф. Заусаев, А. А. Заусаев. Учебное пособие. - Самара: Самарский гос. техн. ун-т, 2006. -88 с.

5. Алтынбаев, Ф.Х. Математическое моделирование движения малых тел Солнечной системы на основе тейлоровских разложений. [Текст]: Автореф. дисс. ... канд. физ.-мат. наук: 05.13 .18 / Ф. Х. Алтынбаев. - Ульяновск, 2005. - 16 с.

6. Волков, E. А. Численные методы [Текст] / Е. А. Волков. - М.: Наука, 1982. - 256 с.

7. Павлова, Г. А. Дифференциальные уравнения. Сборник задач и упражнений [Текст] / Г. А. Павлова. - Самара: СамГТУ, 2005. - 84 с.

Поступила в редакцию $12 / \mathrm{IX} / 2008$;

в окончательном варианте - 30/X/2008.

MSC: 34B99

\section{MODIFIED GRID METHOD FOR SOLVING LINEAR DIFFERENTIAL EQUATION EQUIPPED WITH VARIABLE COEFFICIENTS BASED ON TAYLOR SERIES}

\section{V.P. Radchenko, A. A. Usov}

Samara State Technical University,

443100, Samara, Molodogvardeyskaya str., 244.

E-mail: radch@samgtu.ru

The modified grid method for solving value boundary problems for the linear differential equations based on Taylor development is described. It was demonstrated that the accuracy of the proposed method is much greater than that of a classical grids method. The results of numerical experiments are quoted.

Key words: ordinary differential equations, grid method, Taylor development, closeness, measure of inaccuracy.

Original article submitted 12/IX/2008; revision submitted $30 / \mathrm{X} / 2008$.

Radchenko Vladimir Pavlovich, Dr. Sci. (Phis. E Math.), Prof., Head of Dept. of Applied Mathematics and Computer Science of Samara State Technical University.

Usov Alexandr Alexandrovich, Engineer, Dept. of Higher Mathematics and Applied Computer Science of Samara State Technical University. 